\title{
Signal Quality Checks For Multipath Detection in GNSS
}

\author{
Diego M. Franco-Patiño ${ }^{\# 1}$, Gonzalo Seco-Granados ${ }^{* 2}$, and Fabio Dovis ${ }^{\# 3}$

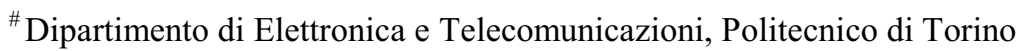 \\ Corso Duca degli Abruzzi, 24, Turin, Italy \\ ${ }^{1}$ ingenierodiegofranco@gmail.com \\ ${ }^{3}$ fabio.dovis@polito.it \\ *Dpt. Telecommunications and Systems Engineering, Universitat Autònoma de Barcelona (UAB) \\ Bellaterra 08193, Barcelona, Spain \\ 22gonzalo.seco@uab.es
}

\begin{abstract}
In GNSS, one of the most important degradations in the signal is caused by the multipath effect, which introduces a bias that impacts the user's position accuracy. The multipath components are attenuated and delayed versions of the transmitted signal, and they are produced by reflections on different obstacles or objects. The aim of this paper is to analyze and compare two multipath detection techniques, namely the Slope Asymmetry Metric (SAM) and the $C / N_{0}$ evolution, as possible tools to check the quality of the signal in different scenarios. It is shown that while both techniques can provide information about the presence of multipath, the SAM is in general more sensitivity to this effect.
\end{abstract}

\section{INTRODUCTION}

The increase of the number of GPS receivers and embedded GPS chipsets in mobile phones was motivated by the need to identify the location of all emergency calls for E-911 and E112 [1], and it had a continuous increasing because this emerging technology is used by the location-based services (LBS) industry. LBS demand a continuous improvement in location accuracy in urban and indoor environments (with very low power signals). But nowadays GPS receivers are also included in mobile phones because they are needed for a large variety of applications.

Strong multipath contributions are usually present in urban environments, which generate perturbations in the incoming signal, making more difficult to obtain an accurate estimation of the receiver's location. Multipath can be detected and mitigated at the antenna level, signal processing level and navigation level. Several different techniques have been implemented in order to detect and mitigate the multipath presence in the signal, as for example, in [2][3] where the multipath components are estimated, in [4] where the multipath effect is detected and mitigated by carrier phase analysis or, in [5] where different methods are proposed for the mitigation of the multipath at the signal-processing level.

The aim of this paper is to analyze at the signal-processing level the quality of the signal in the different scenarios, where there may be presence of multipath using the results obtained from two multipath detection techniques: Slope Asymmetry Metric (SAM) and the carrier-to-noise-spectral-density ratio $\left(C / N_{0}\right)$ evolution. The results of these two techniques will be compared.

\section{Signal Characteristics}

The multipath contribution causes degradation in the signal, and it is constituted by additional replicas of the transmitted signal, which are attenuated, phase shifted and delayed.

Let us assume that the received signal in multipath environments is composed by the Line Of Sight (LOS) signal (the stronger one in most of practical scenarios) plus $M-1$ multipath components, and it is described as:

$$
r(t)=\sum_{i=1}^{M} A_{i}(t) D\left(t-\tau_{i}\right) C\left(t-\tau_{i}\right) e^{j \phi_{i}}+n(t)
$$

where $A_{i}(t)$ is the received amplitude from the $i$-th path, $D(t)$ and $C(t)$ are the transmitted navigation data and C/A code, respectively; $\tau_{i}$ is the propagation delay of the $i$-th path component, $\phi_{i}=2 \pi\left(f_{0}+v_{i}\right) t+\varphi_{i}$ are the received carrier phases of different signal components, $v_{i}$ is the frequency shift, $\varphi_{i}$ is the phase offset and $n(t)$ is the noise.

The multipath combination can be constructive or destructive and it directly affects the correlation peak generated when the incoming signal (from a visible satellite) and the replica C/A code generated in the receiver are correlated. Fig. 1 qualitatively shows this behavior of the correlation function taking in to account just one multipath component.

In this paper three different ways to calculate the correlation are addressed: the first one works in the time domain, and the other two in the frequency domain by using the Fourier transform and the Chirp- $Z$ transform. The last method was the one used for the implementation of the multipath analysis.

\section{MultiPATH DETECTION TECHNIQUES}

\section{A. Slopes Asymmetry of the Correlation Peak}

This multipath detection method compares the left and right slopes of the correlation peak. On the ideal case (nonmultipath, i.e., the blue triangle in Fig. 1), the slopes have the same value but opposite sign. This means that the sum of both slopes should be approximately zero. On the other hand, when multipath is present, one slope is steeper than the other one (red and magenta lines in Fig. 1), and hence the sum of the 
slopes is not close to zero.

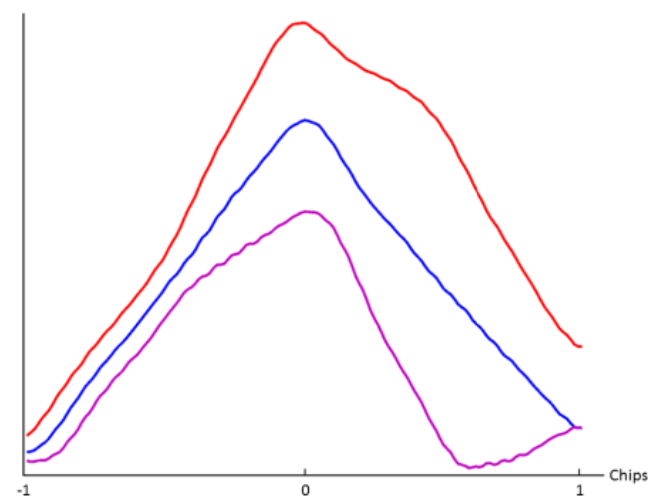

Fig. 1- Correlation peak without multipath (blue line) and with constructive (red) and destructive (magenta) multipath interference.

The sum of the slopes is defined as the Slope Asymmetric Metric (SAM) [6].

$$
S A M=a_{l}+a_{r}
$$

where $a_{l}$ and $a_{r}$ are the estimated slopes of the left and right sides of the correlation triangle, respectively. And they are calculated by fitting 10 points of each side of the correlation curve to a straight line (polynomial of degree 1) by using the polyfit MATLAB function. For this paper the correlation peak is computed using the modulus, in this way it is generated a correlation triangle shape, that is why the sides of the correlation peak are fitted to a polynomial of degree 1 . But there are other possibilities, for example, the square modulus could be also used but the sides of the correlation peak must be fitted to polynomials of degree 2 .

The presence of multipath can be detected starting from the mean $\left(\mu_{S A M}\right)$ and the variance $\left(\sigma_{S A M}\right)$ of the SAM. Thus, if the signal is received in environments with multipath presence, $\mu_{S A M}$ will be different from zero. In static multipath behavior, $\mu_{S A M}$ is nonzero, but the variance is small, whereas in scenarios where the multipath conditions are changing in the interval of time in which the signal was taken, the SAM may vary strongly because of these changes on the multipath conditions. In this latter case, the variance $\sigma_{S A M}$ will present large values, due to the distortion on the correlation triangle produced by constructive and destructive interferences, generating a variability of the SAM (difference of the slopes) larger than the variability produced only by noise.

\section{B. $C / N_{0}$ Evolution}

The signal level suffers variations when the reflections are combined with the LOS signal. The $C / N_{0}$ values can be used to identify different scenarios as described in [6]: $C / N_{0}$ values between 35 and $45(\mathrm{~dB}-\mathrm{Hz})$ correspond to outdoor scenarios, values between 35 and $25(\mathrm{~dB}-\mathrm{Hz})$ to soft indoor such as urban zones and $C / N_{0}$ values less than $25(\mathrm{~dB}-\mathrm{Hz})$ to indoor and deep indoor scenarios.
In multipath scenarios, the signal to noise ratio will change when the multipath conditions are changing, thus the $C / N_{0}$ evolution in time will be an indicator of multipath presence. Here, a higher variance of the $C / N_{0}$ indicates the presence of constructive and destructive interference in the correlation triangle.

In order to analyze the $C / N_{0}$ evolution, the $C / N_{0}$ it is estimated by means of a Narrowband-Wideband Power Ratio (NWPR) method [7], which evaluates the total received power (Signal-plus-Noise Power) of the process over $1 / T_{\text {int }}$ (WideBand Power (WBP)) and 1/(MT int) (Narrow-Band Power (NBP)) noise bandwidths. The expressions of these quantities at the discrete time index $k$ are:

$$
\begin{gathered}
W B P_{k}=\left(\sum_{m=1}^{M}\left(I_{m}^{2}+Q_{m}^{2}\right)\right)_{k} \\
N B P_{k}=\left(\sum_{m=1}^{M} I_{i}\right)_{k}^{2}+\left(\sum_{m=1}^{M} Q_{i}\right)_{k}^{2}
\end{gathered}
$$

where $T_{\text {int }}$ is the integration time, $M$ is the number of samples by navigation bit, and $I$ and $Q$ are the In-Phase and Quadrature prompt correlations, respectively. In the implementation, $T_{\text {int }}$ is 1 millisecond and $M$ is 20 samples.

We can compute:

$$
N P_{k}=\frac{N B P_{k}}{W B P_{k}}
$$

which is a random variable whose statistical mean is estimated as:

$$
\hat{\mu}_{N P}=\frac{1}{K} \sum_{k=1}^{K} N P_{k}
$$

The signal to noise ratio estimation, in $\mathrm{dB}-\mathrm{Hz}$ units, is:

$$
\frac{\widehat{C}}{N_{0}}=10 \log _{10}\left(\frac{1}{T_{\text {int }}} \frac{\hat{\mu}_{N P}-1}{M-\hat{\mu}_{N P}}\right)
$$

\section{EXPERIMENTAL SCENARIOS}

In order to analyze the changes on the signal due to the presence of multipath, several streams of signal samples have been collected in 3 different scenarios. In the first, residential outdoor scenario, the receiver is placed in a small prominence with some buildings at one of its sides (Zone 0 in Fig. 2), having good visibility of the satellites (its skyplot is shown in Fig. 3). Canyon Scenario is the second one and it is shown in Fig. 2 as "Zone1". In this case the receiver is placed in between buildings, each of them has a height about 15 meters and they are separated approximately 20 meters from the point where the receiver antenna is placed to acquire the signal; the skyplot of this scenario is shown in Fig. 4. And in the third one the signal is captured while the receiver pass from "Zone 0" (Residential Outdoor Scenario) to "Zone1" (Canyon 
Scenario); the skyplot of the detected satellites is shown in Fig. 5.

These scenarios were carefully preselected, in this way, the only thing that could change between one point and another was the presence or absence of multipath. The measurements were taken in a small area, so it is not realistic to assume that different interference conditions were present along the trajectory. Moreover, there were no trees, and changes in the antenna gain are not likely to have occurred since the duration of the signal captures was 37 seconds.

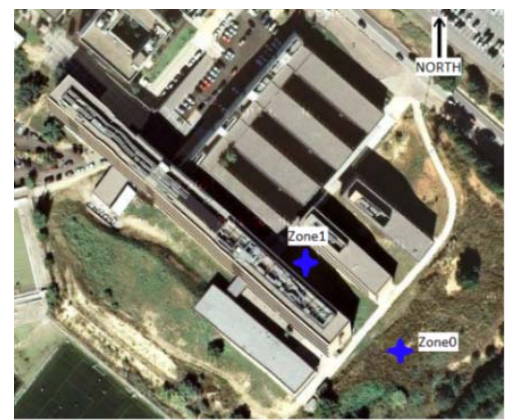

Fig. 2 - Places (blue X) where the raw signals were taken: Residential outdoor (Zone0) and Canyon (Zone1) scenarios.
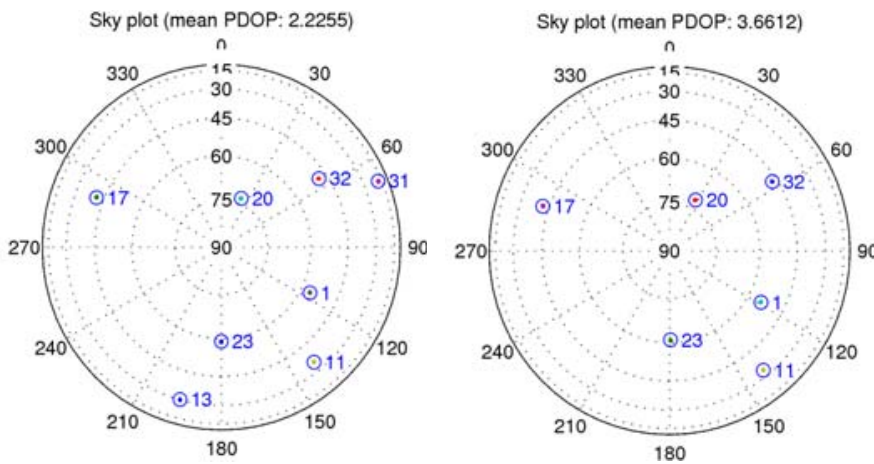

Fig. 3 - Skyplot: Residential Outdoor Scenario

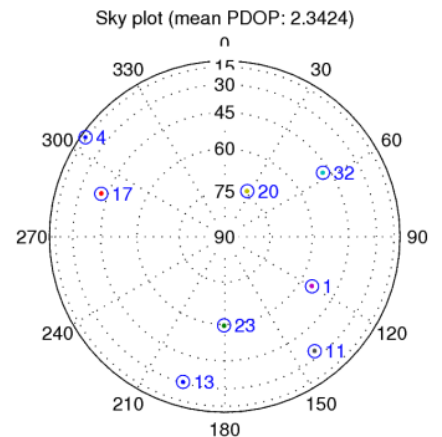

Fig. 5 - Skyplot: Walking from Residential Outdoor to Canyon Scenario

\section{RESULTS}

Raw GPS signals samples were acquired during 37 seconds with the "GN3S Sampler v2" front-end and they are processed by means of the MATLAB code included in [8] with some modifications.

\section{A. Correlation Execution Time}

The three methods used to calculate the correlation give the same outputs but the execution time of each one is different. Fig. 6 shows that the Fourier transform is the faster (lowest execution time), while the Chirp- $Z$ transform is the slower one (highest execution time).

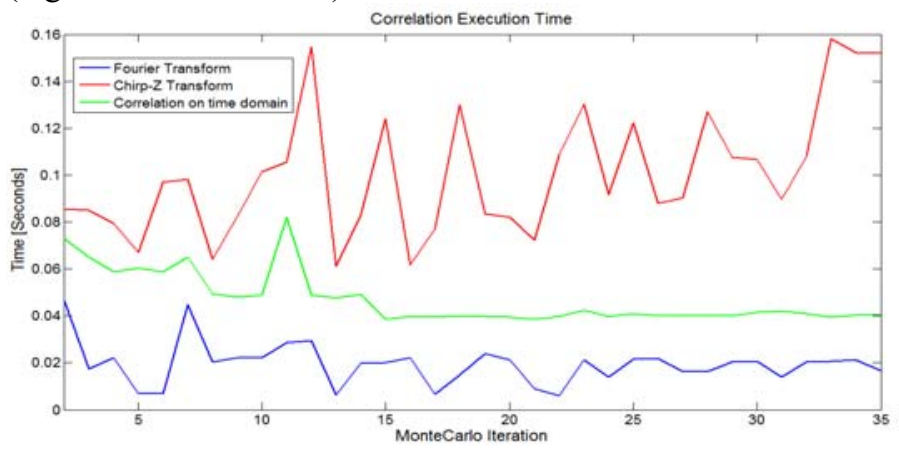

Fig. 6 - Correlation Execution Time. FFT-Blue, Chirp-Z-Red and Time domain-Green

\section{B. Slopes Asymmetry Analysis}

In absence of multipath, the SAM should be take values around zero, but in the implementation it was found that it had values around -52 in every satellite. The specific value is not relevant because its units depend on the implementation of the signal processing algorithms. The relevant aspect is that the SAM in the absence of multipath is clearly not null, revealing an asymmetry in the correlation peak. Thus, in this work, in the ideal case (i.e. multipath-free) the SAM metric is not taken as zero, but -52 . The filter in the front-end causes this effect by generating a correlation triangle that is not completely symmetrical unlike it is usually assumed. This happens because the filter impulse response has not perfect symmetry around its maximum.

As mentioned above, the mean and the standard deviation of the SAM indicate multipath presence in a scenario. The mean and the standard deviation are calculated during sliding windows of duration equal to 3 seconds.

- $\quad$ Residential Outdoor Scenario (Zone0 in Fig. 2, skyplot in Fig. 3)

Fig. 7 shows the behavior of the SAM metric obtained from the modulus of the correlation peak for signal interval equal to one bit. In this case most of the signals of different satellites take values around -52 , indicating good visibility of the detected satellites.

Fig. 8 shows the SAM standard deviations, which oscillate around 40 for all the satellites, which indicates, the contribution of the constructive and destructive interferences has not large variations. These values of the standard deviation are due to the noise.

- $\quad$ Canyon Scenario(Zone1 in Fig. 2, skyplot in Fig. 4)

In this case the behavior of the SAM metric hints at the presence of multipath in some satellites (Fig. 9). For example, SV20 (brown line), with high elevation and hence low signal reflections, has SAM values close to those considered as multipath absence (-52), whereas SV23 (mustard line), with 
lower inclination and hence higher signal reflections, has values around -90 , indicating stronger multipath presence in the signal.

The standard deviation (shown in Fig. 10) takes values between 30 and 60 for all the satellites, which is a larger range than in outdoor scenario, indicating the presence of constructive and destructive multipath combinations. In this case, SV17 has higher values from 5 to 15 seconds and from 20 to 25 seconds, reflecting stronger multipath contribution than the other satellites.

- Walking from Residential outdoor to Canyon scenario (from Zone 0 to Zone 1, skyplot Fig. 5)

The skyplot in Fig. 5 shows the visible satellites in the Zone 0 position, that is, the point where the signal samples were started to be captured.

Fig. 11 shows the SAM metric behavior where, during the first 15 seconds, the values are more or less constant, but from the second 15 onwards, the SAM takes values a bit greater (as SV4-cyan line) or lower (as SV23-mustard line). This happens because the buildings generate multipath interference in the signal when the receiver is in Zone 1. The SAM metric of SV13 (blue line) experiences a strong change between the seconds 15 and 20; this happens because the satellite has low elevation and the signal is blocked by a building when the receiver goes in between the buildings.

The standard deviations of all satellites take values between 30 and 55 (Fig. 12), indicating that the constructive and destructive interferences are more or less the same in the different signals, and they are similar to the ones in the canyon scenario case.

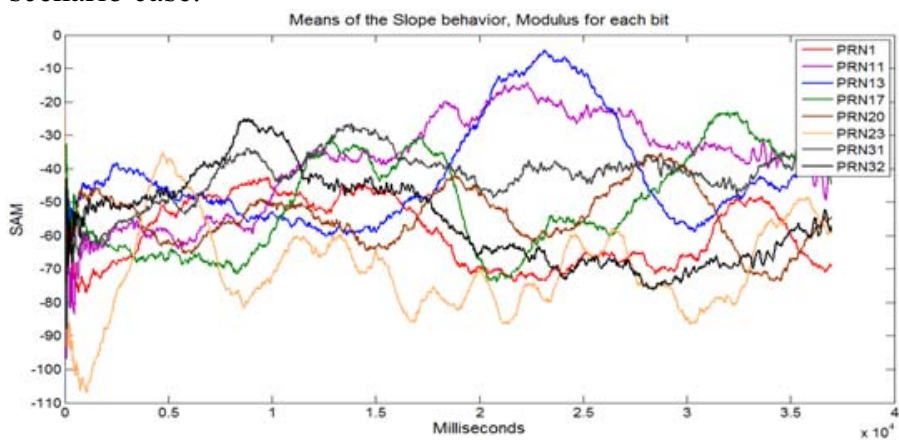

Fig. 7 - Behavior of the SAM sliding mean (Outdoor residential scenario).

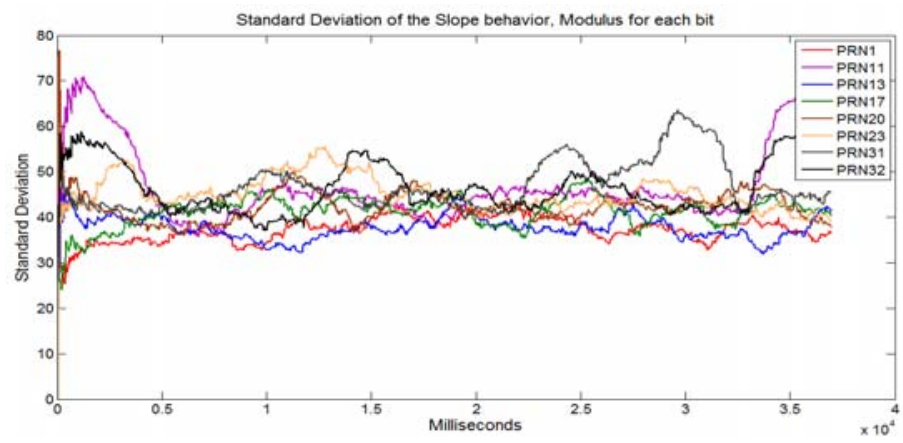

Fig. 8 - Behavior of the Standard Deviation of the SAM (Outdoor residential scenario).

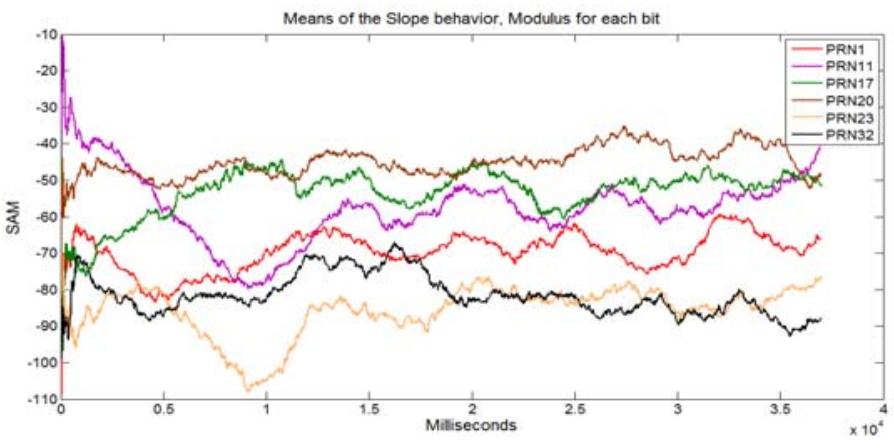

Fig. 9 - Behavior of the SAM sliding mean (Canyon scenario).

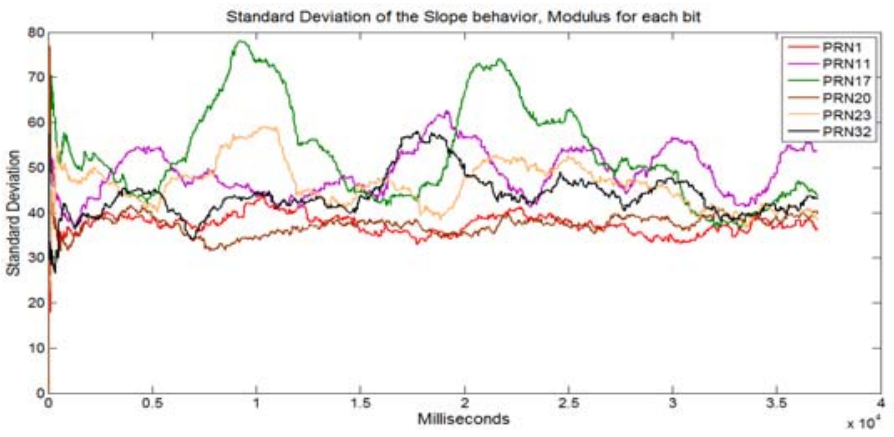

Fig. 10 - Behavior of the Standard Deviation of the SAM (Canyon scenario).

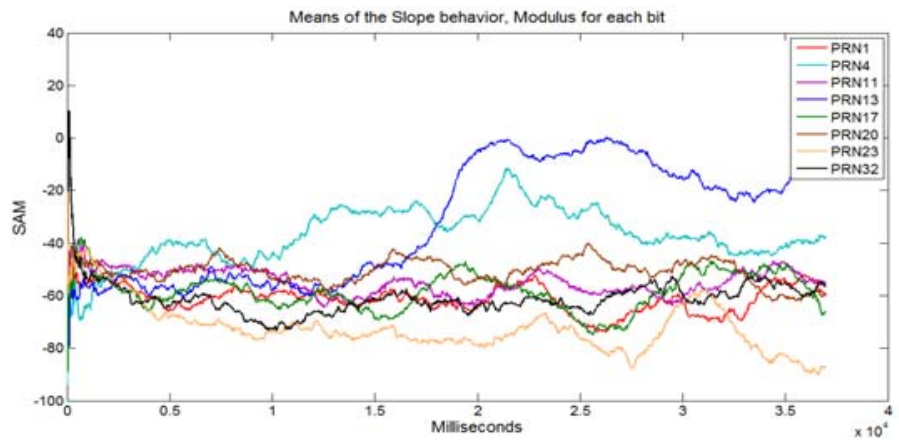

Fig. 11 - Behavior of the SAM sliding mean (Walking from Residential outdoor to Canyon scenario).

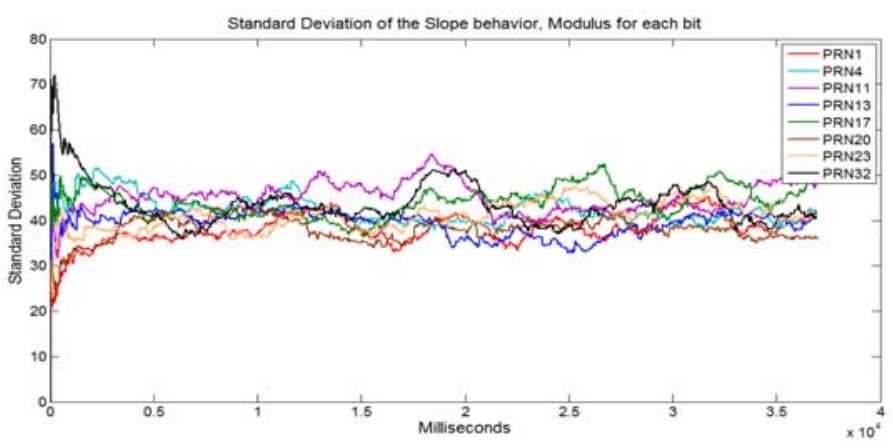

Fig. 12 - Behavior of the Standard Deviation of the SAM (Walking from Residential outdoor to Canyon scenario).

\section{C. $C / N_{0}$ Evolution Analysis}

The $C / N_{0}$ mean values and the standard deviation of the $C / N_{0}$ are calculated in order to analyze the $C / N_{0}$ evolution with respect to the time, and detect the presence of multipath. $C / N_{0}$ values are computed by taking $K=1$ in ( 6) and a 
sliding windows of 3 seconds.

- $\quad$ Residential Outdoor Scenario (Zone 0 in Fig. 2, skyplot in Fig. 3)

In this scenario there are 8 satellites in view, and almost of them have $C / N_{0}$ mean values larger than $40 \mathrm{~dB}-\mathrm{Hz}$ (Fig. 13), while two of them (SV13 and SV11) take values between 35 and $40 \mathrm{~dB}-\mathrm{Hz}$. All of these are considered normal values for outdoor scenarios [6] and do not present big variations along the whole period of time.

In Fig. 14 the standard deviations of the $C / N_{0}$ values are shown considering the whole period of time, and all of them are constant on the time, even if they have different values. In this case, the SV13, SV11 and SV32 present the highest standard deviations while the other ones take values approximately around 1 . In particular, the fact that SV13 and SV11 present lower $C / N_{0}$ values with higher standard deviation seems to be related with the variation of the SAM in Fig. 7.

- $\quad$ Canyon Scenario (Zone 1 in Fig. 2, skyplot in Fig. 4)

In this case the signals from 6 satellites were acquired, 5 of which have $C / N_{0}$ larger than or equal to $35 \mathrm{~dB}-\mathrm{Hz}$ (see Fig. 15). These values are usually experienced in outdoor scenarios, and just one (SV11) has values slightly smaller than $35 \mathrm{~dB}-\mathrm{Hz}$, which is a value usually present in more dense urban scenarios [6].

Fig. 16 shows the standard deviation of the $C / N_{0}$ variation with respect to the $C / N_{0}$ sliding mean. The three satellites that present the lowest $C / N_{0}$ values have a standard deviation higher than the ones with the higher $C / N_{0}$ values.

The satellites with the lowest $C / N_{0}$ and highest standard deviation present an unusual and unexpected behavior between the 20 and 30 seconds, which could be related with a burst of ones presented in the bits of the navigation message during this period of time for each of these satellites.

- Walking from Residential outdoor to Canyon scenario (from Zone 0 to Zone 1, skyplot Fig. 5)

In Fig. 17 the $C / N_{0}$ sliding mean from the 8 different satellites detected is shown. SV4, that is the satellite with the lowest elevation, presents the lowest $C / N_{0}$ values, which are around $20 \mathrm{~dB}-\mathrm{Hz}$, considered as a value usually corresponding to an indoor scenario [6]. SV11 and SV20 have values approximately of $35 \mathrm{~dB}-\mathrm{Hz}$, considered as urban residential scenario. Furthermore the other 5 satellites present values greater than $40 \mathrm{~dB}-\mathrm{Hz}$, found usually in outdoor scenarios, and it indicates good visibility of the satellites.

The SV13 presents good values of the $C / N_{0}$ until the second 15 , where it starts to decrease, because the signal from this satellite is blocked by a building as was also highlighted in the analysis of the SAM. The behavior in this zone can be compared with the strong change in the SAM in Fig. 11. The $C / N_{0}$ of this satellite begins to recover after second 27 until obtaining stable values around $35 \mathrm{~dB}-\mathrm{Hz}$. This last received signal is surely obtained from Non-line-of-sight (NLOS) reflection, because, as was previously stated, the SV13 signal is blocked.

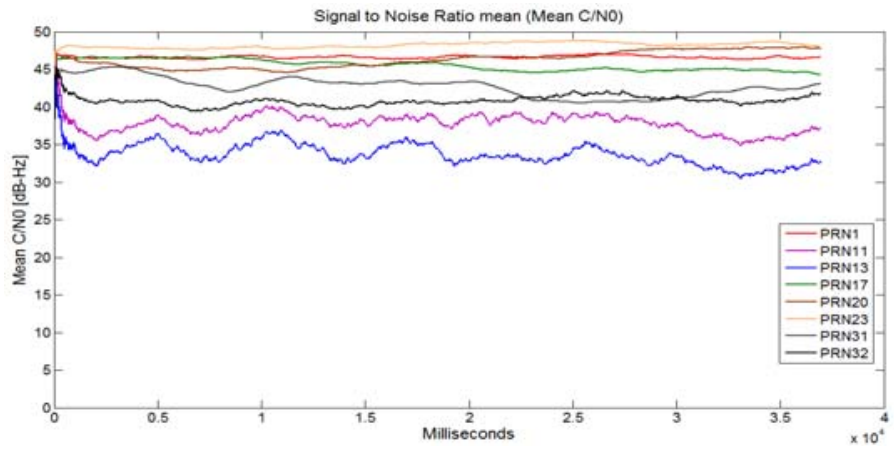

Fig. $13-C / N_{0}$ sliding mean. $K=1$ (outdoor residential scenario)

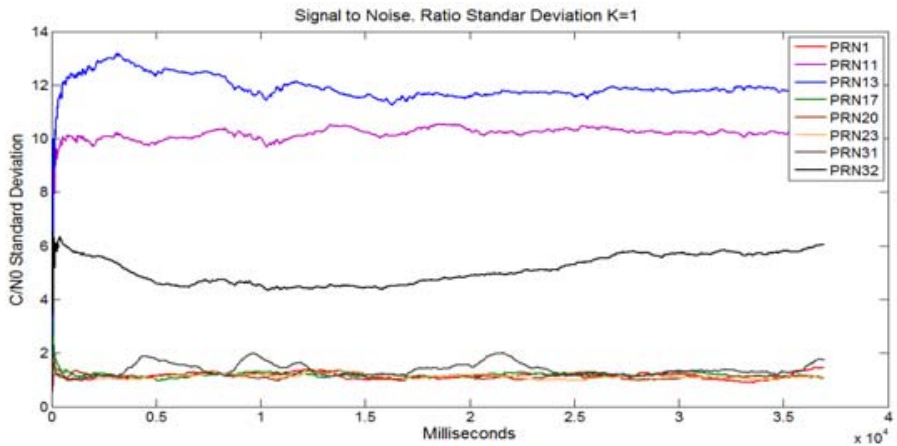

Fig. 14 - Standard deviation of the $\boldsymbol{C} / \boldsymbol{N}_{\mathbf{0}}$ variation with respect to the $\boldsymbol{C} / \boldsymbol{N}_{\mathbf{0}}$ mean. $K=1$ (outdoor residential scenario)

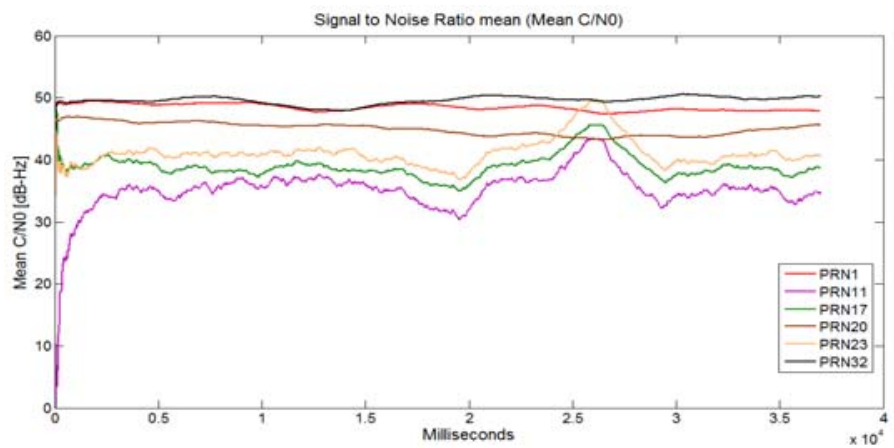

Fig. 15 - $\boldsymbol{C} / \boldsymbol{N}_{\mathbf{0}}$ sliding mean. $K=1$. (Canyon Scenario)

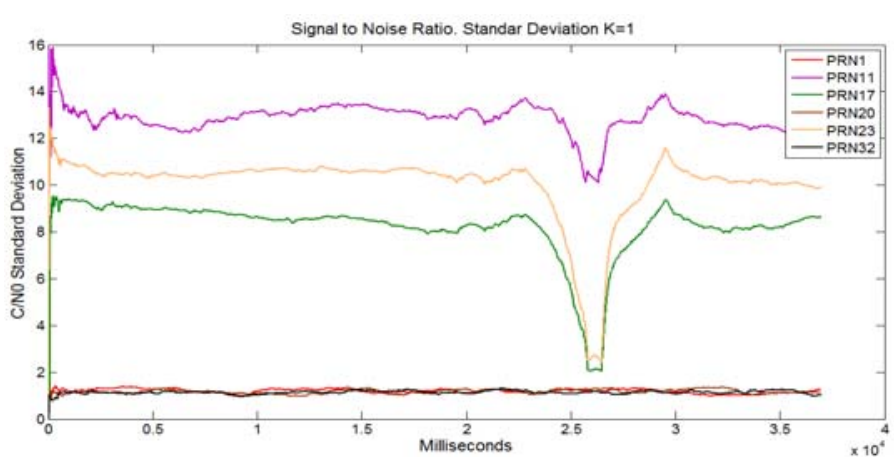

Fig. 16 - Standard deviation of the $\boldsymbol{C} / \boldsymbol{N}_{\mathbf{0}}$ variation with respect to the $\boldsymbol{C} / \boldsymbol{N}_{\mathbf{0}}$ mean. $K=1$. (Canyon Scenario) 


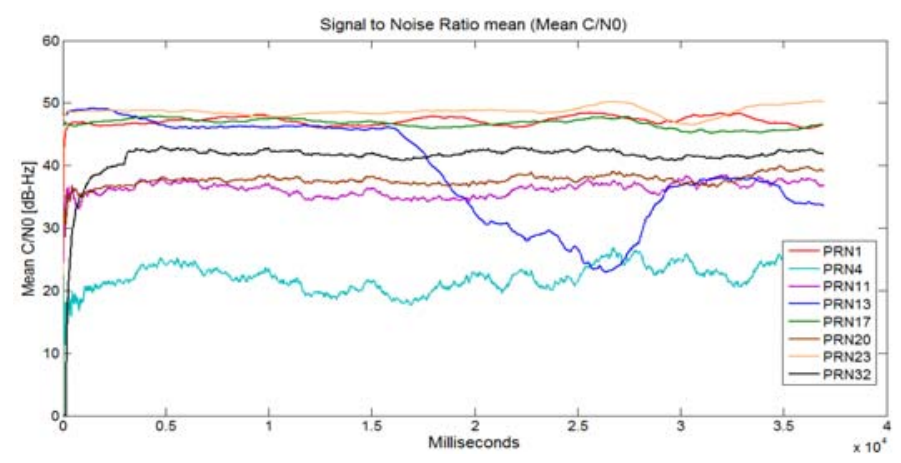

Fig. $17-C / N_{0}$ sliding mean. $K=1$. (Walking from outdoor residential to Canyon Scenario)

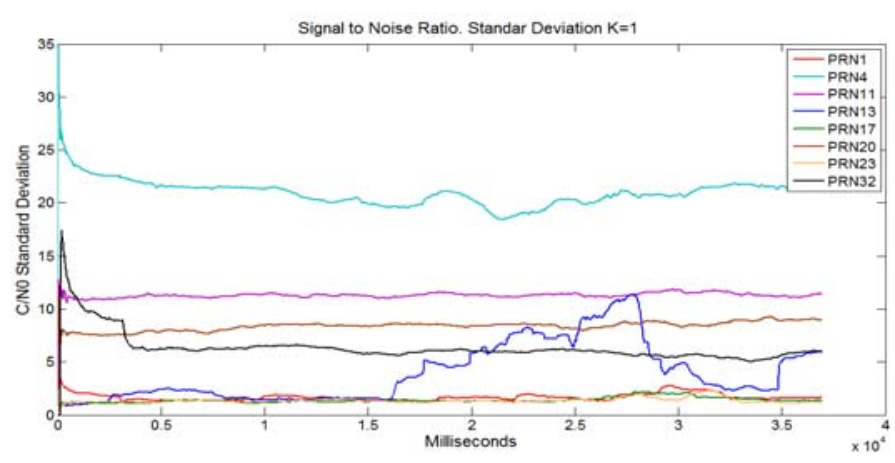

Fig. 18 - Standard deviation of the $\boldsymbol{C} / \boldsymbol{N}_{\mathbf{0}}$ variation with respect to the $\boldsymbol{C} / \boldsymbol{N}_{\mathbf{0}}$ mean. $K=1$. (Walking from outdoor residential to Canyon Scenario)

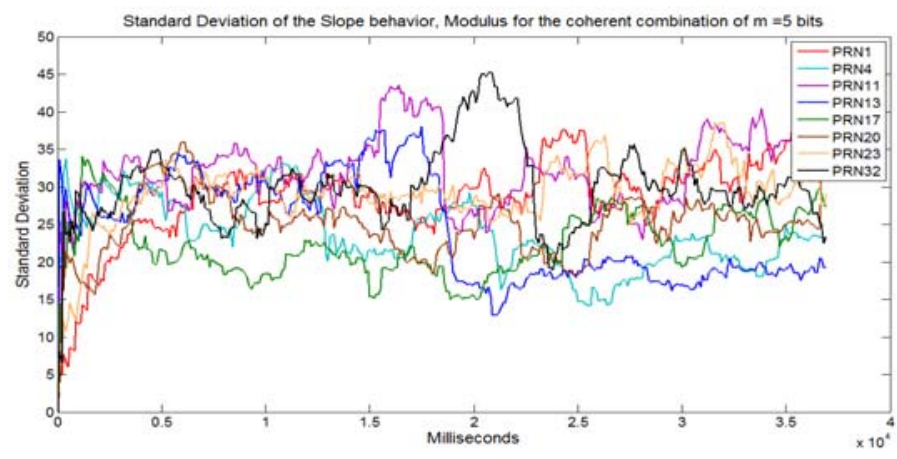

Fig. 19 - Standard deviation of the SAM values, doing the modulus for the coherent combination of $m=5$ bits

At this point is also important to consider the behavior of the SV4 which presents the lowest but almost constant $C / N_{0}$ values in all the period, while in this scenario the multipath conditions change in time. At the other hands, using the SAM technique (Fig. 11), this satellite presents soft but considerable variation over time, clearer showing its variation when the multipath is present.

The standard deviation of the $C / N_{0}$ variation with respect to the $C / N_{0}$ sliding mean is shown in Fig. 18, where all the satellites, also even if they have different values, take values that are approximately constant during the whole period of time, with the exception of the signal coming from the satellite 13 which, again, presents a variation starting approximately from the second 15 (moment in which the signal is blocked by a building); this variations indicate constructive and destructive multipath contribution variation.

As shown in Fig. 12, the SAM standard deviation does not present clearly this effect for the SV 13. But doing coherent combinations of 5 bits (Fig. 19) this effect becomes clearer.

\section{CONCLUSIONS}

In this paper two different techniques for multipath detection have been analyzed. Different approaches to compute the correlation function necessary for the both techniques have been compared in terms of complexity. The Fourier transform has smaller execution time in the calculation of the correlation than the time domain and the Chirp-Z transform approaches.

It was shown that calculating slopes by using the modulus of the correlation is appropriate to analyze the asymmetry of the correlation curve based on the metric named SAM. The $C / N_{0}$ estimation in each bit (without doing coherent combination of bits) is also appropriate to analyze the multipath presence. The SAM technique is considered more promising than analyzing the $C / N_{0}$ evolution, because it shows clearer multipath changes.

\section{ACKNOWLEDGEMENTS}

The work of G. Seco-Granados was supported in part by the Spanish Ministry of Economy and Competitiveness project TEC 2011-28219.

\section{REFERENCES}

[1] FCC, "Revision of the Commission's Rules to Ensure Compatibility with Enhanced 911 Emergency Calling Systems", Docket No. 94-102, United States Federal Communications Commission, 1996.

[2] B. Townsend, R. D. J. van Nee, P. Fenton, and K. J. van Dierendonck, "Performance Evaluation of the Multipath Estimating Delay Lock Loop", Proc. ION National Technical Meeting, 1995.

[3] X. Chen, F. Dovis, M. Pini, P. Mulassano, "Turbo architecture for multipath mitigation in global navigation satellite system receivers," Radar, Sonar \& Navigation, IET , vol.5, no.5, pp.517,527, June 2011

[4] O. M. Mubarak, A. Dempster "Carrier phase analysis to mitigate multipath effect", International Global Navigation Satellite Systems Society, IGNSS Symposium, 2007.

[5] K.Benachenhou, E. Sari, M.Hammadouche, "Multipath Mitigation in GPS/Galileo Receivers with Different Signal Processing Techniques", 5th International Conference: Sciences of Electronic, Technologies of Information and Telecommunications, March 2009.

[6] J. A. López-Salcedo, J. M. Parro-Jiménez, G. Seco-Granados, "Multipath detection metrics and attenuation analysis using a GPS snapshot receiver in harsh environments", Antennas and Propagation, 2009. EuCAP 2009. 3rd European Conference on, vol., no., pp.36923696, 23-27 March 2009.

[7] B. W. Parkinson, J. J. Spilker, "Global Positioning System: Theory and Applications", American Institute of Aeronautics and Astronautics, vol. $1,1996$.

[8] K. Borre, D.M.Akos, N. Bertelsen, P. Rinder, S.H. Jensen, " $A$ Software-Defined GPS and Galileo Receiver A Single-Frequency Approach", Birkhauser, Boston, 2007. 